\title{
ENTORNOS VIRTUALES DE ENSEÑANZA-APRENDIZAJE. EVALUACIÓN DEL USO DE LAS HERRAMIENTAS VIRTUALES EN EL MÁSTER DE EDUCACIÓN PARA EL DESARROLLO
}

\author{
VIRTUAL ENVIRONMENTS FOR TEACHING-LEARNING. \\ EVALUATION OF THE USE OF VIRTUAL TOOLS IN THE MASTER OF EDUCATION \\ FOR SUSTAINABLE DEVELOPMENT \\ Luisa Torres Barzabal; $\underline{\text { barzabal@upo.es }}$ \\ Esther Prieto Jiménez; eprijim@upo.es \\ Luis López Catalán; luislopcat@upo.es \\ Universidad Pablo de Olavide de Sevilla
}

\begin{abstract}
RESUMEN
El uso de las plataformas virtuales como recurso docente en el ámbito universitario, es una realidad cada vez más generalizada. A lo largo de este artículo realizaremos una evaluación de la puesta en marcha del Máster Universitario en Educación para el Desarrollo (EpD), implementado en la Universidad Pablo de Olavide de Sevilla, y realizado on-line en su totalidad. Todo ello lo llevaremos a cabo con el análisis de los datos extraídos del curso académico 2009-2010.
\end{abstract}

PALABRAS CLAVE: Educación, desarrollo, plataformas, entornos virtuales, e-learning, herramientas, recursos.

\section{ABSTRACT}

The use of virtual platforms as a teaching resource in the university, is an increasingly common reality. Throughout this article we will evaluate the implementation of the Master's Degree in Education for Development (EPD), implemented at the University Pablo de Olavide de Sevilla, and performed on-line in its entirety. This will make it to the analysis of data from the 2009-2010 academic year.

KEYWORD: Education, development, platforms, virtual environments, e-learning, tools, resources. 


\section{INTRODUCCIÓN}

La sociedad de la información y del conocimiento ha cambiado nuestro estilo de vida en todos sus ámbitos, es por ello que también ha cambiado nuestro procedimiento de enseñar y de aprender, para adaptarnos, como afirma Fandos (2006), a las necesidades de la sociedad actual que lleva un ritmo acelerado y que crece a escala global (Castells, 2010). Uno de estos cambios, más relevantes, es la posibilidad de su desarrollo en la modalidad de formación virtual mediante plataformas educativas.

Actualmente se está despertando en todas las Universidades Andaluzas un alto interés por la incorporación de las TIC, de forma general, y las redes telemáticas de forma específica, como concluyen Ballesteros, Cabero, Llorente y Morales (2010:12), por lo que es cada vez más frecuente encontrar cursos, congresos, másters, etc., que se ofertan con la modalidad e-learning o blearning.

La formación virtual, además de considerar ventajas competitivas, como afirman Bergiel, Bergiel y Balsmeier (2008), se fundamenta principalmente en posibilitar formación a un mayor número de personas que en la formación presencial, a menor coste, sin límite geográfico o de horario, personalizando el aprendizaje, donde la innovación, la generación de conocimientos y la velocidad de respuesta, entre otros, son cruciales; generando lo que algunos autores han denominado como un nuevo paradigma de enseñanza-aprendizaje.

Sin lugar a dudas, uno de los elementos fundamentales para el óptimo desarrollo de la formación virtual son las herramientas de e-learning y la utilización que de ellas se realice, ya que compartimos con Cabero (2006:8) que no serán los determinantes técnicos del sistema los que marcarán su calidad y su eficacia, sino la atención que les prestemos a las variables educativas y didácticas que se ponen en funcionamiento, por ello, previamente a la consecución de las competencias que se pretenden alcanzar en dicho encuentro educativo, será necesario desarrollar el aprendizaje autónomo y el correcto uso de las tecnologías de la información y comunicación.

Quisiéramos señalar que el buen uso de las nuevas tecnologías de la información para la docencia, es sin duda una opción ventajosa, tanto para el profesorado como para el alumnado; no obstante, aunque la habilidad y desenvoltura de las herramientas requiere de un periodo de adaptación y práctica, las dificultades pueden ser superadas con la planificación explicita del uso de la plataforma y la valoración del esfuerzo continuo requerido del alumnado. Pero es fundamental que el objetivo final, lejos del mero instrumentalismo, sea el uso de estas herramientas como medios para la construcción compartida del conocimiento en comunidades de aprendizaje (Lave y Wenger, 2003), en el que pueda desarrollarse los indicadores de calidad presentados por Ardilla (2011).

En línea con lo antes comentado, a continuación nos adentramos en la descripción y características del Máster de Educación para el Desarrollo (EpD). 


\section{EL MÁSTER DE EDUCACIÓN PARA EL DESARROLLO (EPD). METODOLOGÍA}

Con la intención de describir y conocer, de una manera más específica, el contexto en el que se desarrolla la plataforma virtual, a la que estamos haciendo alusión a lo largo de este escrito, y que compartimos con Gil y Roca (2011) que se trata de una herramienta necesaria para la formación universitaria europea, presentamos, seguidamente, una serie de apartados que contribuirán a su ubicación, tanto en el contenido sobre el que versa, como una explicación detallada de las herramientas que la conforman.

\subsection{Breve descripción y características del Máster de EpD}

El Máster de Educación para el Desarrollo, Sensibilización Social y Cultura Social, se crea en la Universidad Pablo de Olvide de Sevilla en el curso académico 2008-2009, como título propio de dicha institución.

El fundamento conceptual sobre el que se sustenta esta titulación y que, a su vez argumenta su relevancia en la oferta universitaria, es la consideración de la Educación para el Desarrollo como una propuesta de comprensión global de la humanidad (Osler: 1994).

Diferentes Declaraciones, Planes y Documentos internacionales, nacionales y autonómicos, asientan las bases esenciales que justifican la creación y desarrollo de este título en el ámbito universitario. Concretamente podemos aludir a la Declaración Universal de los Derechos Humanos del año 1948, la cual indica en su artículo 26 la necesidad de impartir una docencia con la finalidad última de alcanzar el pleno desarrollo de la persona:

"La educación tendrá por objeto el pleno desarrollo de la personalidad humana y el fortalecimiento del respeto a los derechos humanos y a las libertades fundamentales; favorecerá la comprensión, la tolerancia y la amistad entre todas las naciones y todos los grupos étnicos o religiosos (...)".

Seguidamente podemos mencionar la Declaración del Milenio (firmada en el año 2000) en la que se recogen las intenciones, a nivel internacional, en la que se establecen 8 objetivos y 18 metas a alcanzar antes del año 2015, relacionadas con el desarrollo y la reducción de la pobreza.

Centrándonos en el ámbito universitario, queremos hacer referencia a la situación actual de reforma en la que se encuentra inmersa la institución universitaria con el proceso de convergencia en el Espacio Europeo de Educación Superior. Concretamente, en el comunicado de Bergen (2005, p. 5) se cita lo siguiente:

"Nuestra contribución para conseguir la educación para todos debe basarse en el principio de desarrollo sostenible y estar de acuerdo con el trabajo internacional en curso sobre el desarrollo de directrices para garantizar la calidad de la educación superior transnacional. Reiteramos que en la cooperación académica internacional deben prevalecer los valores académicos". 
Atendiendo a lo anteriormente expuesto y conforme a Duart $(2001,1)$, con el propósito de sumar conocimiento, experiencia, reflexión, análisis, etc., queda de manifiesto la necesidad de la creación e implementación de este máster universitario que tiene como finalidad (CEDEP, 2009:21):

- Formar expertos en fundamentos, metodologías y competencias propias de la ED

- Diseñar didácticamente planes, programas, proyectos y acciones de ED.

- Mejorar el rendimiento de equipos de ED desde el análisis y evaluación de los mismos.

- Adquirir los fundamentos, herramientas y destrezas para la identificación, diseño, seguimiento y evaluación de calidad de planes, programas y proyectos de Educación para el Desarrollo tanto a nivel general como a nivel de formación de formadores.

- Obtener un conocimiento de la situación de la Educación para el Desarrollo a nivel internacional desde el conocimiento y estudio de Buenas Prácticas.

- Participar en estudios e investigaciones sobre ED en España, Europa y América Latina.

- Promover una capacitación suficiente para realizar una memoria académica y tendrán un adecuado marco de referencia para colaborar en las tareas técnicas propias de la Educación para el Desarrollo tanto en el ámbito público como privado.

- Ofrecer líneas de investigación en ED para nuevos estudios y espacios de diseño e intervención.

En cuanto a las características esenciales del alumnado que ha participado en la realización de dicho curso, mediante la modalidad a distancia, hemos de aludir que fueron un total de 35 estudiantes los que cursaron este máster durante esta edición. De entre el total de alumnado, indicamos que procedían, principalmente de ciudades andaluzas y españolas, aunque hemos de destacar a estudiantes de otros países, también de habla hispana, como México y Perú y, en relación a la formación previa del alumnado matriculado, el abanico de posibilidades aumenta, contando entre otros, esencialmente, a titulados en las ramas de la ciencias sociales (fundamentalmente ciencias de la educación, educación social, trabajo social...) aunque también han participado en él titulados en periodismo, derecho, etc.

Habiendo justificado la pertinencia y necesidad de contar con un título de postgrado centrado en la Educación para el Desarrollo, la Sensibilización Social y la Cultura Social, pasamos a analizar la plataforma virtual en la que se desarrolló la segunda edición de este master on-line, en el curso académico 2009-2010.

\subsection{Descripción de la modalidad virtual que ofrece, así como de la plataforma usada}

El Máster de Educación para el Desarrollo curso 2009-10, opta por desarrollarse en la modalidad virtual, por considerar que esta circunstancia tiene una serie de ventajas para la formación, ya que es un medio ideal para la información, comunicación y participación de los miembros de la 
comunidad educativa, pues no se necesita el desplazamiento físico, lo que conlleva menores costes y tiempos invertidos. De igual forma, da la posibilidad de formarse en cualquier horario y con flexibilidad de tiempo dedicado al aprendizaje, así como de compaginar el trabajo, las tareas domésticas... con la formación y además, proporciona el espacio adecuado para el desarrollo de la actividad académica, con los recursos de que dispone y procurando un aprendizaje individualizado, es decir adaptado al ritmo de cada persona.

Actualmente podemos encontrar diferentes entornos virtuales para la enseñanza; como Moodle, ILIAS, Blackboard, Virtual Profe, Bazaar (Sánzhez Rodríguez, 2009: 221-222). Las diferentes plataformas virtuales con una finalidad educativa que hallamos en la actualidad, podemos clasificarlas en aquellas que son de libre distribución y las comerciales, tal y como nos indican Ramos, De la Osa y De Toro en la siguiente tabla:

\begin{tabular}{|c|c|}
\hline $\begin{array}{c}\text { LIBRES } \\
\text { (OPEN SOURCE) }\end{array}$ & $\begin{array}{c}\text { NO LIBRES } \\
\text { (COMERCIALES) }\end{array}$ \\
\hline ATutor & Angel \\
\hline Bodington & Authorware \\
\hline Dokeos & Blackboard \\
\hline KEWL & Desiee2Learn \\
\hline .LRN & Edumate \\
\hline LON-CAPA & Knowlwdgw Forum \\
\hline Moodle & Scholar360 \\
\hline Sakai Project & WebCT \\
\hline LogiCampus & Litmos \\
\hline SWAD & CyberExtension \\
\hline
\end{tabular}

Tabla 1. Plataformas Virtuales Educativas.

Fuente: Adaptación de Ramos, De la Osa y De Toro (2009:6-7)

De entre todas las plataformas virtuales destinadas a la educación, la Universidad Pablo de Olavide de Sevilla hace uso de la WebCT, es por ello que el Máster de EpD, al ser un título ofertado por esta institución, se desarrolla en la citada plataforma.

La Universidad Pablo de Olavide, de Sevilla toma la iniciativa en España poniendo en el entorno de explotación la última versión disponible de la Plataforma de Docencia Virtual WebCT como Apoyo Tecnológico a la Innovación Académica y es la plataforma que utilizamos en dicho Máster.

A continuación presentamos las herramientas que se emplearon en el espacio virtual del Máster de Educación para el Desarrollo durante el curso 2009-10.

\subsection{Los diferentes recursos utilizados en dicha plataforma}

En este epígrafe se dará a conocer las principales funcionalidades de las diferentes herramientas de la plataforma WebCT en el Máster de EpD curso 2009-10. 
El diseño, organización y actualización del espacio virtual en WebCT fue responsabilidad del profesor/a encargado/a del curso, por lo que no todos los módulos presentaron la misma estructura.

Las herramientas que el alumno/a encontró en su espacio WebCT son de organización (buscar, calendario y programa), de comunicación (anuncio, conversación, correo, foro de debate, lista y usuarios en línea), de actividades de aprendizaje (evaluaciones y tareas), de contenido (biblioteca de medios, módulos de aprendizaje y vínculos web) y del alumno/a (mis calificaciones, mi progreso y notas).

Los espacios que el alumno/a pudo encontrar en su espacio WebCT son los que se muestran en la figura siguiente.

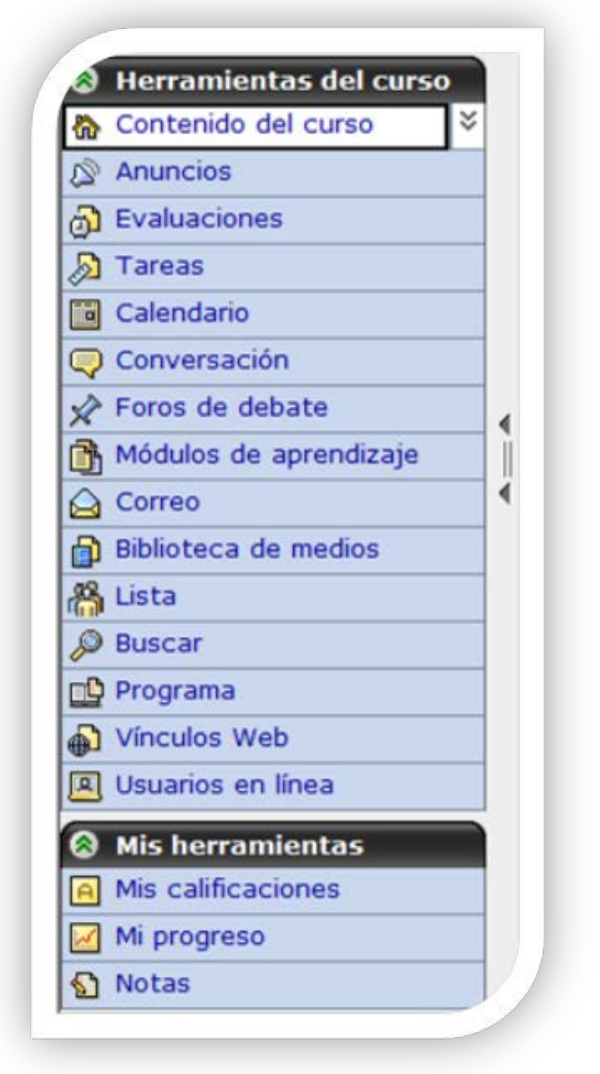

Figura 1: Herramientas del curso

A continuación describimos brevemente las posibilidades de cada una de ellas que les ayudará a entretejer las redes de interacción como señalan García y Suárez (2011).

- Contenidos del curso: pertenece a la página principal en la que se muestran diferentes carpetas y documentos (archivos) para distribuir el contenido de cada uno de los módulos que conforman el Máster de EpD. Estos documentos presentados en html se corresponden con el temario del curso e información en relación a ellos. 
- Anuncios: en esta ubicación se publicaron todas las noticias que los profesores/as consideraron relevantes para el grupo.

- Evaluaciones: los profesores/as pudieron evaluar los conocimientos del alumnado a través de cuestionarios o exámenes, también se incluyeron autoevaluaciones para que el alumnado comprobara su comprensión de la temática.

- Cuestionarios: con ellos las respuestas son anónimas, sólo le sirvió al profesorado para tener un conocimiento general sobre un tema.

- Exámenes: los exámenes permitieron evaluar los conocimientos de manera automática y fijar una calificación, que si el profesor/a lo permitió, el alumnado pudo visualizar.

- Autoevaluaciones: son exámenes que le permitió al alumnado evaluar sus conocimientos de manera automática, pero no almacenar sus resultados. Sirvió para que practicaran los conocimientos adquiridos.

- Tareas: además de las evaluaciones, también se solicitaron tareas individuales o en grupo. Mediante esta herramienta "TAREAS", el profesorado comunicó al alumnado los trabajos que tuvieron que realizar, y éste también fue el espacio para que lo enviaran una vez realizados.

- Calendario: Se usó para mostrar las citas (tutorías, chat, comienzo de módulos, fechas para entrega de tareas, etc.) y plazos puestos por los profesores/as, así como se invitó a marcar las del propio alumnado.

- Conversación: la pizarra electrónica permitió conversar en tiempo real, así como mostrar imágenes, dibujar objetos, insertar gráficos,... Resultó especialmente útil para dibujar y visualizar diagramas en tiempo real. Se habilitaron diferentes espacios o salas en función de las temáticas y módulos.

- Foros de debate: el foro de debate fue muy útil y usado para discutir sobre los diferentes temas, quedando todas las opiniones registradas, pudiendo consultarlas en cualquier momento.

- Se abrieron espacios para cada módulo y dentro de ellos se establecieron diferentes categorías, o temas y en cada tema se incluyeron los comentarios correspondientes a él, intentando respuestas anidadas.

- Correo: esta herramienta el alumnado la utilizó para comunicarse con los profesores/as o los compañeros/as de manera asincrónica.

- Biblioteca de medios: este espacio contiene las definiciones de las palabras claves en la asignatura. Un glosario de términos.

- Lista: en este apartado se incluyó los perfiles de los miembros del curso (profesores/as y alumnos/as). Consideramos que era importante que todos/as editaran su propio perfil, con fotografía e información relevante para el máster. 
- Buscar: se ofrece para buscar en el contenido del curso. Permitiendo acotar la búsqueda mediante área, seleccionando la herramienta, la fecha...

- Programa: en este sitio se alojó el programa completo del máster de EpD.

- Vínculos Web: en este espacio se incluyeron los vínculos web que se consideraron importantes para completar la materia de cada uno de los módulos que componen el Máster.

- Usuarios en línea: el chat permitió mantener conversaciones en tiempo real con todas las personas conectadas al Sistema de Aprendizaje.

- Mis calificaciones: en este espacio el alumnado pudo ver las calificaciones que iba obteniendo en los distintos exámenes o tareas realizadas en cada módulo de forma privada. Estas calificaciones no fueron únicamente numéricas, también incluían comentarios a sus trabajos.

- Mi progreso: en este apartado el alumnado pudo efectuar un seguimiento de su propio progreso, pudiendo ver las secciones del curso a la que accedía y el número de páginas de contenidos visitadas.

- Notas: la aplicación permitió al alumnado que tomase notas, ideas, reflexiones,... en su propio cuaderno. Estas notas pudieron utilizarlas o imprimirlas en cualquier momento, así como eliminarlas.

Presentadas las herramientas a disposición del alumnado, seguidamente nos adentramos en la evaluación que sobre el uso de ellas han realizado en el Máster de EpD 2009-10.

\section{3. eVAluación del uso que hizo el alumnado de la plataforma en el MÁSTER DE EPD. CURSO 2009-2010. RESULTADOS.}

Entendemos que para alcanzar una mejora en el desarrollo e implementación del máster en la plataforma virtual WebCT, es preciso realizar una revisión de su uso y aplicación durante un curso académico. Coincidimos con González, Acosta y Moyares (2010:5) cuando indican que "evaluar la usabilidad y accesibilidad de una aplicación web es ventajoso, pues descubrir qué errores de diseño tiene la web es el primer paso para poder corregirlos".

Centrándonos en analizar el uso de la plataforma en la que se desarrolló este máster, procedemos a la presentación e interpretación de los datos más relevantes obtenidos a lo largo del curso 20092010.

Concretamente aludiremos a aquellas cuestiones que describen los aspectos más significativos para la evaluación que planteamos sobre el uso de la WebCT que realizó el alumnado: tiempo empleado en la plataforma, secciones más visitadas, etc.

En primer término presentamos la siguiente tabla en la que recogemos una serie de datos generales sobre el uso de la plataforma, incidiendo en el número total de horas empleadas a lo largo del curso, la media de conexión en las sesiones, media de sesiones por alumnado en el 
desarrollo del máster (días laborables y fines de semana); así como apreciaremos la hora de más actividad en la plataforma y aquella, que menos usuarios registró.

\begin{tabular}{|lc|}
\hline \multicolumn{1}{|c|}{ Indicadores } & Valor \\
\hline Número total de sesiones de usuario & 2593 \\
\hline Media de tiempo dentro de las sesiones de usuario & $00: 17: 21$ \\
\hline Media de sesiones de usuario por día & 25 \\
\hline Media de sesiones de usuario por día en fines de semana & 0,28 \\
\hline Media de sesiones de usuario por día en días de diario & 16 \\
\hline Hora del día más activa & $13: 00$ - 14:00 \\
\hline Hora del día menos activa & $06: 00$ - 07:00 \\
\hline
\end{tabular}

Tabla 2: Indicadores sobre el uso de la plataforma.

Tal y como podemos contemplar en la tabla anterior, a lo largo del curso académico 2009-2010, se desarrollaron un total de 2.593 sesiones en la WebCT del máster de Educación para el Desarrollo, repartidas entre los meses de diciembre de 2009 a diciembre de 2010; con una media de 17 minutos y 21 segundos por sesión. En la siguiente figura se muestran las sesiones empleadas por cada estudiante.

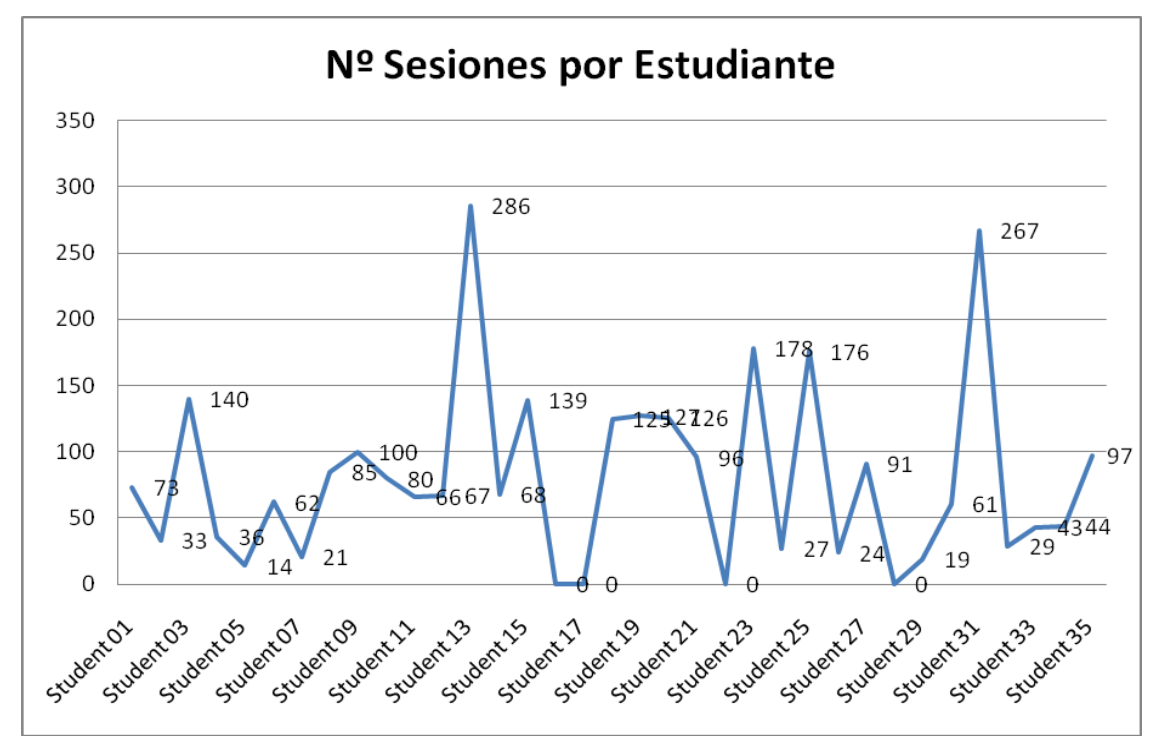

Figura 2: Número de sesiones de cada Estudiante al Máster de EpD

La media de sesiones por día a lo largo del curso se centró en torno a 25 , siendo mucho más frecuentada la plataforma durante los días laborable (16 sesiones) en contraposición a los fines de semana $(0,28)$.

Finalmente, hacemos referencia a la hora del día en la que más alumnado se conectaba a la plataforma virtual, centrándose ésta entre las 13:00h y 14:00 (hora española); por el contrario, la franja horaria menos activa se concentra entre las 06:00h y las 07:00h. 
Atendiendo a la evaluación del uso de cada una de las herramientas que componen la plataforma, presentamos seguidamente una tabla en la que se recopilan el número de sesiones, la media de tiempo por sesión, el tiempo total y el porcentaje de sesiones totales.

\begin{tabular}{|lcccc|}
\hline \multicolumn{1}{|c}{ Herramientas } & Sesiones & $\begin{array}{c}\text { Media de tiempo por } \\
\text { sesión }\end{array}$ & $\begin{array}{c}\text { Tiempo } \\
\text { total }\end{array}$ & $\begin{array}{c}\text { Porcentaje de } \\
\text { sesiones totales }\end{array}$ \\
\hline $\begin{array}{l}\text { Administrador de } \\
\text { archivos }\end{array}$ & 114 & $0: 00: 59^{1}$ & $1: 51: 57$ & $0.56 \%$ \\
\hline Anuncios & 638 & $0: 00: 33$ & $5: 53: 26$ & $1.76 \%$ \\
\hline Archivo & 2744 & $0: 02: 21$ & $107: 26: 13$ & $\mathbf{3 2 . 0 7 \%}$ \\
\hline Biblioteca de medios & 93 & $0: 04: 27$ & $6: 54: 11$ & $2.06 \%$ \\
\hline Buscar & 44 & $0: 00: 45$ & $0: 33: 18$ & $0.17 \%$ \\
\hline Calendario & 766 & $0: 00: 47$ & $9: 54: 12$ & $2.96 \%$ \\
\hline Carpeta & 5671 & $0: 00: 25$ & $39: 12: 47$ & $11.70 \%$ \\
\hline Conversación & 494 & $0: 00: 55$ & $7: 29: 14$ & $2.23 \%$ \\
\hline Correo & 1162 & $0: 00: 54$ & $17: 26: 43$ & $5.21 \%$ \\
\hline Evaluaciones & 68 & $0: 03: 15$ & $3: 40: 49$ & $1.10 \%$ \\
\hline Foros de debate & 2988 & $0: 01: 10$ & $58: 14: 57$ & $17.39 \%$ \\
\hline Mis calificaciones & 571 & $0: 00: 25$ & $4: 00: 48$ & $1.20 \%$ \\
\hline Notas & 218 & $0: 00: 38$ & $2: 19: 24$ & $0.69 \%$ \\
\hline Seguimiento & 295 & $0: 00: 40$ & $3: 14: 52$ & $0.97 \%$ \\
\hline Tareas & 1163 & $0: 01: 29$ & $28: 45: 41$ & $8.58 \%$ \\
\hline Usuarios en línea & 609 & $0: 01: 01$ & $10: 23: 25$ & $3.10 \%$ \\
\hline Vista imprimible & 357 & $0: 04: 11$ & $24: 51: 59$ & $7.42 \%$ \\
\hline Vínculos Web & 106 & $0: 01: 35$ & $2: 47: 23$ & $0.83 \%$ \\
\hline Total & 18101 & $0: 26: 30$ & $335: 01: 19$ & $100.00 \%$ \\
\hline
\end{tabular}

Tabla 3: Tiempo de utilización de las herramientas

Consultados los datos planteados apreciamos que la herramienta más utilizada por el alumnado (32.07\%) se centra en el "archivo", utilizada en 2.744 ocasiones, con una media de 2 minutos y 21 segundo, haciendo un total de 107 horas, con 26 minutos y 13 segundos.

La segunda de las herramientas más frecuentadas es el "Foro de Debate" (17,39\%). En esta ocasión se han contabilizado 2.988 sesiones en las que se ha utilizado, empleando una media de tiempo de 1 minuto con 10 segundos por cada una de ellas, alcanzando una cifra total de 58 horas con 14 minutos y 57 segundos, en las que el alumnado participó en los foros de debate.

\footnotetext{
${ }^{1}$ Los tiempos aparecen en horas, minutos y segundos.
} 
En la siguiente tabla mostramos los datos correspondientes a cada uno de los alumnos/as del Máster de EpD.

\begin{tabular}{|c|c|c|c|}
\hline Alumnos/as & Sesiones & Tiempo total & Foros de debate \\
\hline Student 01 & 73 & $19: 28: 27$ & 277 \\
\hline Student 02 & 33 & $11: 17: 37$ & 169 \\
\hline Student 03 & 140 & 71:54:04 & 818 \\
\hline Student 04 & 36 & $26: 13: 42$ & 104 \\
\hline Student 05 & 14 & $8: 03: 31$ & 230 \\
\hline Student 06 & 62 & 27:06:04 & 805 \\
\hline Student 07 & 21 & $9: 29: 23$ & 192 \\
\hline Student 08 & 85 & 29:50:03 & 854 \\
\hline Student 09 & 100 & $43: 26: 33$ & 822 \\
\hline Student 10 & 80 & $32: 17: 59$ & 902 \\
\hline Student 11 & 66 & $17: 54: 26$ & 547 \\
\hline Student 12 & 67 & $29: 25: 07$ & 370 \\
\hline Student 13 & 286 & $18: 24: 04$ & 231 \\
\hline Student 14 & 68 & $18: 21: 06$ & 815 \\
\hline Student 15 & 139 & $16: 51: 48$ & 817 \\
\hline Student 16 & 0 & 0:00:00 & 0 \\
\hline Student 17 & 0 & 0:00:00 & 0 \\
\hline Student 18 & 125 & $43: 38: 33$ & 454 \\
\hline Student 19 & 127 & $17: 55: 42$ & 160 \\
\hline Student 20 & 126 & $40: 51: 38$ & 939 \\
\hline Student 21 & 96 & $30: 56: 13$ & 756 \\
\hline Student 22 & 0 & 0:00:00 & 0 \\
\hline Student 23 & 178 & $63: 24: 44$ & 812 \\
\hline Student 24 & 27 & 7:17:02 & 180 \\
\hline Student 25 & 176 & $63: 46: 22$ & 1196 \\
\hline Student 26 & 24 & $7: 14: 55$ & 145 \\
\hline Student 27 & 91 & $13: 59: 57$ & 673 \\
\hline Student 28 & 0 & 0:00:00 & 0 \\
\hline Student 29 & 19 & 7:44:16 & 52 \\
\hline Student 30 & 61 & $19: 32: 46$ & 477 \\
\hline Student 31 & 267 & $34: 26: 30$ & 962 \\
\hline Student 32 & 29 & $7: 26: 56$ & 313 \\
\hline Student 33 & 43 & 19:16:10 & 279 \\
\hline Student 34 & 44 & $20: 45: 54$ & 613 \\
\hline Student 35 & 97 & $46: 41: 17$ & 652 \\
\hline Total & 2514 & $806: 38: 45$ & 16385 \\
\hline
\end{tabular}

Tabla 4: Tiempo del alumnado en los foros de debate 
En la tabla de datos anterior, apreciamos como de los 35 alumnos/as que han participado en este curso, hay estudiantes que han participado en más de 1.000 sesiones de los foros de debates, mientras que en la otra cara de la moneda contamos con aquellos que han participado en 100 sesiones, dejando al margen a los que no se han conectado a esta herramienta a lo largo del curso.

Como resumen de lo expuesto han sido más de 806 horas las que el alumnado ha empleado en la participación de los foros de debates, bien iniciados por el profesorado implicado, como por los mismos compañeros/as, llegando a producirse 16.385 participaciones en los mismos.

Finalmente, como hemos presentado en la tabla 2, aludimos a aquella herramienta que menos uso ha tenido por parte del alumnado $(0,17 \%)$ que se materializa en el apartado "buscar". En esta ocasión sólo ha sido utilizada en 44 sesiones, con un tiempo total de 33 minutos y 18 segundos, lo que indica que el tiempo medio por sesión ha sido de 45 segundos.

Como se ha podido apreciar las herramientas de contenido son las más usadas por el alumnado. Esta cuestión puede deberse a que es en esta sección en la que el alumnado encontraba la mayoría del contenido a trabajar y sobre el que versaba el temario del máster. Dentro de las herramientas de contenido se hallan los vínculos web, las carpetas de contenido y archivos.

\section{Herramientas de contenido}

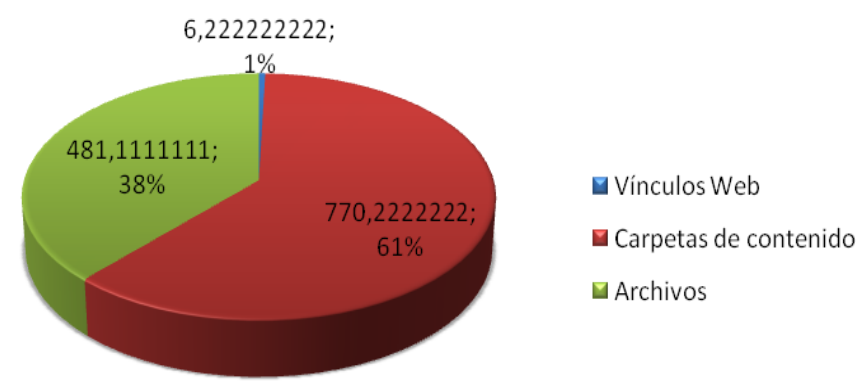

Figura 3: Herramientas de contenido

Confirmando la idea que argumentábamos antes de la presentación de la figura 3, son las carpetas de contenido, en la que se ubicaban los textos esenciales de los módulos que componen el temario de este máster, la herramienta más frecuentada por el alumnado. Por el contrario, los vínculos web se establecen como el apartado menos frecuentado en esta sección.

Del mismo modo y de forma general, son muy utilizadas las herramientas de comunicación, por esta cuestión, posteriormente detallamos el uso realizado por los alumnos/as para el correo electrónico en la plataforma WebCT, el foro de debate y el chat: 
Correo electrónico:

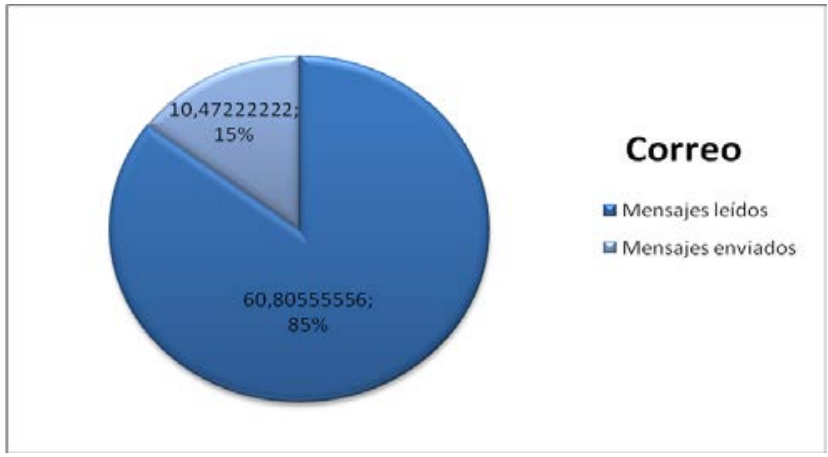

Figura 4: Uso del correo electrónico de la WebCT

El porcentaje es mucho mayor respecto de los mensajes que el alumnado lee que los que envía, pudiéndose entender que el uso que realiza del foro es principalmente para recibir información.

\section{Foro de debate:}

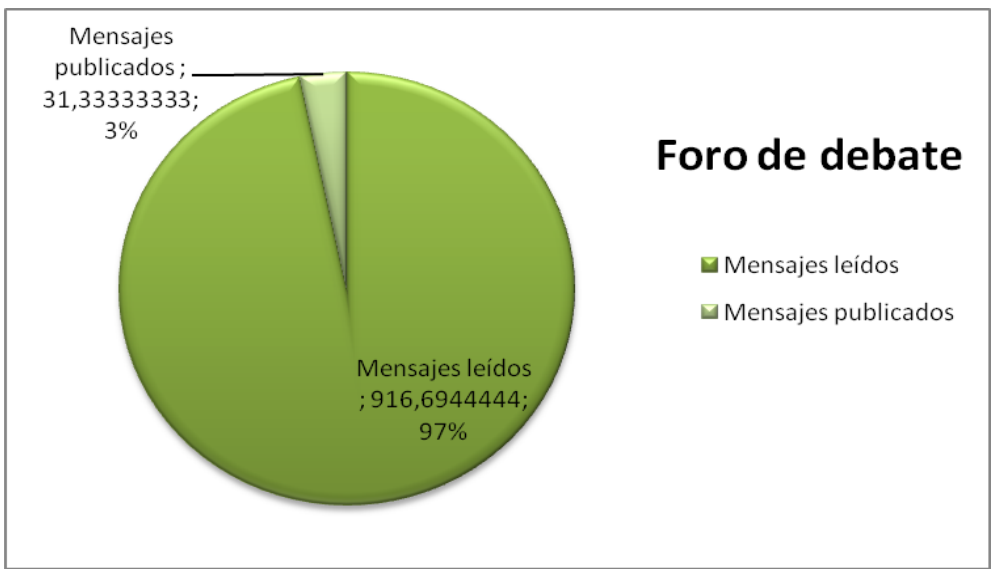

Figura 5: Uso del foro de debate de la WebCT

De igual modo puede apreciarse que el foro es leído en un porcentaje mayor a los publicados, no obstante se aprecia que el porcentaje de mensajes publicados $31,3 \%$, se muestra muy equilibrado al número de alumnos/as que cursan el máster en dicha edición. 


\section{Chat}

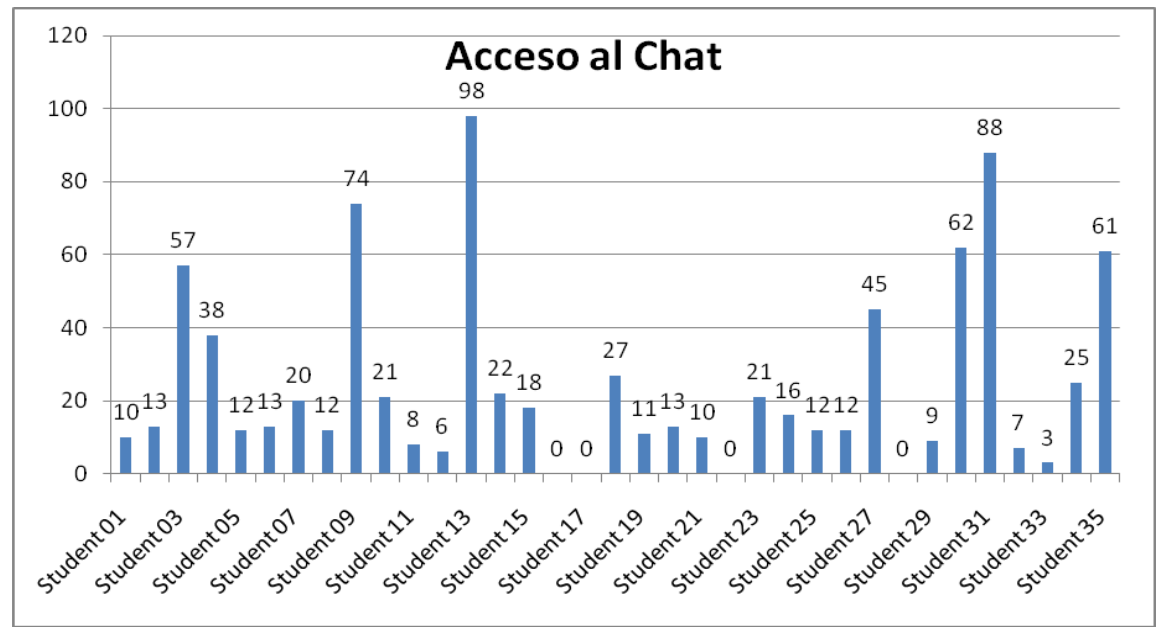

Figura 6: Acceso al chat de la WebCT

Salvo dos estudiantes, todos han tenido acceso al chat en mayor o menor medida, despuntando mucho el número de entrada de algunos estudiantes sobre otros.

La evaluación que se les realizó al alumnado respecto a los contenidos del Máster de EpD 2009-10, fue mediante test y tareas y respecto al uso que de éstas herramientas de evaluación han realizado el alumnado podemos apreciar los siguientes datos sobre ellas:

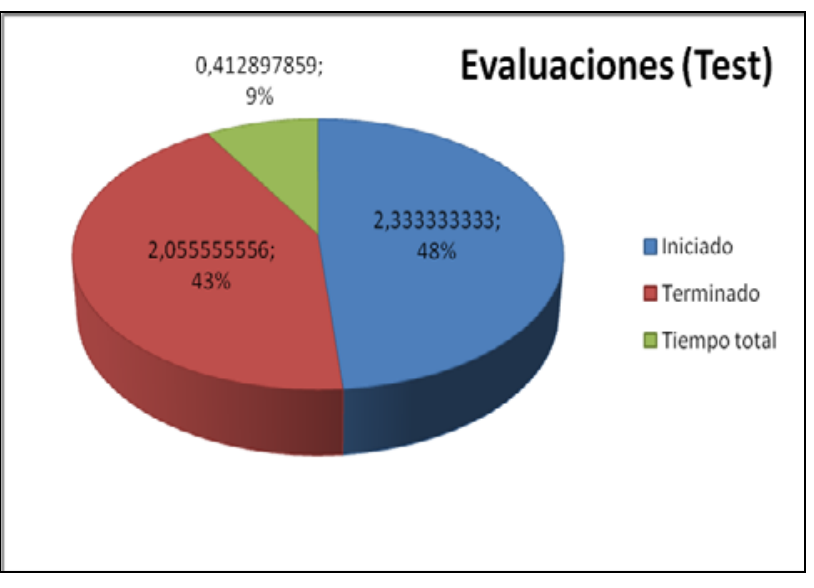

Figura 7: Evaluación (Test)

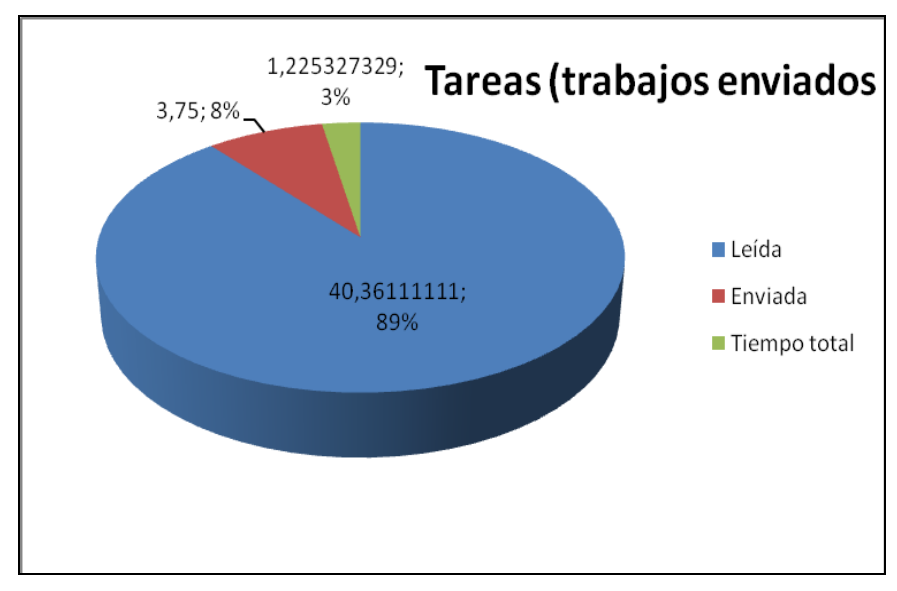

Figura 8: Tareas (trabajos enviados) 


\begin{tabular}{|c|c|c|c|c|c|c|}
\hline \multirow[t]{2}{*}{ Alumnos/as } & \multicolumn{3}{|c|}{ Evaluaciones (test) } & \multicolumn{3}{|c|}{ Tareas (trabajos enviados) } \\
\hline & Iniciado & Terminado & $\begin{array}{c}\text { Tiempo } \\
\text { total }\end{array}$ & Leída & Enviada & $\begin{array}{c}\text { Tiempo } \\
\text { total }\end{array}$ \\
\hline Student 01 & 1 & 0 & $0: 23: 42$ & 15 & 2 & $5: 32: 36$ \\
\hline Student 02 & 0 & 0 & 0:00:00 & 9 & 1 & $14: 29: 24$ \\
\hline Student 03 & 2 & 2 & $1: 58: 26$ & 49 & 3 & $28: 33: 57$ \\
\hline Student 04 & 1 & 0 & $1: 42: 40$ & 20 & 2 & $8: 27: 48$ \\
\hline Student 05 & 1 & 1 & $5: 21: 17$ & 16 & 2 & 28:51:37 \\
\hline Student 06 & 2 & 2 & $2: 17: 09$ & 26 & 3 & $1: 45: 18$ \\
\hline Student 07 & 0 & 0 & 0:00:00 & 2 & 0 & $0: 43: 12$ \\
\hline Student 08 & 1 & 1 & $3: 17: 36$ & 31 & 3 & $6: 30: 28$ \\
\hline Student 09 & 1 & 1 & $0: 43: 28$ & 48 & 3 & $17: 48: 22$ \\
\hline Student 10 & 3 & 3 & $8: 40: 44$ & 37 & 3 & $2: 19: 48$ \\
\hline Student 11 & 3 & 3 & $12: 36: 49$ & 48 & 2 & $12: 50: 11$ \\
\hline Student 12 & 1 & 0 & $0: 03: 16$ & 13 & 1 & $29: 49: 50$ \\
\hline Student 13 & 6 & 4 & $3: 11: 30$ & 61 & 3 & $27: 21: 05$ \\
\hline Student 14 & 4 & 4 & $8: 34: 20$ & 38 & 4 & $36: 35: 19$ \\
\hline Student 15 & 4 & 4 & $16: 18: 49$ & 20 & 2 & $36: 35: 00$ \\
\hline Student 16 & 0 & 0 & & 0 & 0 & \\
\hline Student 17 & 0 & 0 & & 0 & 0 & \\
\hline Student 18 & 1 & 0 & $6: 12: 05$ & 32 & 3 & $8: 20: 24$ \\
\hline Student 19 & 0 & 0 & 0:00:00 & 23 & 4 & 7:07:30 \\
\hline Student 20 & 1 & 1 & 31:33:00 & 26 & 3 & $40: 21: 20$ \\
\hline Student 21 & 1 & 1 & 9:03:33 & 11 & 1 & $10: 03: 25$ \\
\hline Student 22 & 0 & 0 & & 0 & 0 & \\
\hline Student 23 & 1 & 1 & $9: 28: 22$ & 29 & 4 & $32: 38: 47$ \\
\hline Student 24 & 0 & 0 & 0:00:00 & 3 & 0 & 1:06:18 \\
\hline Student 25 & 2 & 2 & $11: 45: 06$ & 34 & 2 & $46: 55: 17$ \\
\hline Student 26 & 1 & 1 & $5: 42: 54$ & 20 & 1 & $0: 23: 56$ \\
\hline Student 27 & 0 & 0 & 0:00:00 & 14 & 2 & $4: 33: 37$ \\
\hline Student 28 & 0 & 0 & & 0 & 0 & \\
\hline Student 29 & 0 & 0 & 0:00:00 & 7 & 0 & $4: 47: 57$ \\
\hline Student 30 & 0 & 0 & 0:00:00 & 23 & 2 & $38: 20: 57$ \\
\hline Student 31 & 2 & 2 & $8: 29: 16$ & 46 & 4 & $6: 34: 46$ \\
\hline Student 32 & 0 & 0 & 0:00:00 & 10 & 2 & $2: 57: 37$ \\
\hline Student 33 & 0 & 0 & 0:00:00 & 15 & 3 & $3: 17: 24$ \\
\hline Student 34 & 0 & 0 & 0:00:00 & 9 & 0 & $0: 32: 03$ \\
\hline Student 35 & 6 & 6 & $12: 44: 53$ & 22 & 4 & $17: 56: 52$ \\
\hline Total & 39 & 35 & $156: 57: 25$ & 696 & 66 & 456:51:00 \\
\hline Media & 2,33333333 & 2,05555556 & 0,41289786 & 40,3611111 & 3,75 & 1,22532733 \\
\hline
\end{tabular}

Tabla 5: Evaluaciones 
Analizando ya el último de los aspectos que presentamos en este artículo, centraremos nuestra atención en las dos herramientas que el alumnado ha utilizado para ser evaluado virtualmente en el Máster de EpD.

Atendiendo a los datos presentados apreciamos que los estudiantes del máster han utilizado los test de autoevaluación con una media de 41 minutos cada uno; mientras que en el caso de la herramienta "tareas" esta media temporal asciende a 1 hora y 22 minutos. De ello se concluye que en la utilización de las herramientas de evaluación, son las tareas en las que más tiempo emplea el alumnado.

Otro de los aspectos a resaltar es que determinados alumnos/as han utilizado la herramienta de tareas, pero no han usado en ningún momento de su participación en el máster, los tests de evaluación; esta cuestión se debe a que los test presentados en esta edición eran de autoevaluación, por lo que no tenían un carácter obligatorio, sólo pretendía orientar al alumnado acerca de su nivel de conocimiento sobre el contenido estudiado.

En el caso de la tarea, sí que era de uso obligatorio, ya que se utilizaba como la vía para hacer llegar al equipo docente el trabajo final de cada materia, que conformaba el peso esencial de la calificación final de cada módulo.

Hemos realizado una aproximación y descripción esencial sobre la formación on-line del Máster de EpD y el uso de la plataforma virtual de este máster en el curso 2009-10 y a continuación quisiéramos extraer algunas conclusiones.

\section{PRESENTACIÓN DE LAS CONCLUSIONES.}

Las nuevas tendencias en el ámbito educativo están derivando fronteras en lo que respecta a la presencialidad y el proceso de enseñanza-aprendizaje en un lugar determinado en unas fechas y horas inamovibles. En este sentido presentamos el ejemplo del Máster de Educación para el Desarrollo, título propio de la Universidad Pablo de Olavide de Sevilla, llevado a cabo de manera on-line.

Tras analizar las principales herramientas de la plataforma virtual en la que se ha desarrollado este máster, desde la perspectiva del alumnado participante, presentamos seguidamente las principales conclusiones que hemos obtenido.

Hemos de decir que a pesar de ser éste un máster bajo la modalidad virtual de enseñanza aprendizaje, en el que cada alumno y alumna puede decidir el momento y el tiempo que destina libremente para su realización, llama la atención cómo la gran mayoría de los estudiantes suelen trabajar en horario laboral, durante la semana, siendo la participación los fines de semana menos activa.

En cuanto al manejo de los contenidos, se ha comprobado que aún teniéndolos organizados de una manera más didáctica en la plataforma, el alumnado sigue prefiriendo descargarse los 
archivos y documentos alojados en la plataforma (en formato pdf), en lugar de acceder a más información mediante los enlaces y vínculos web que se les facilita.

Las herramientas de comunicación son esenciales en la modalidad virtual, ya que mediante ellas el alumnado se siente más seguro y acompañado en el proceso de enseñanza-aprendizaje, tanto por el equipo docente como por el resto de los compañeros/as.

Aludiendo a las herramientas de comunicación, es el foro de debate la más frecuentada por el alumnado. En estos foros el debate entre compañeros/as ha sido muy rico, tanto para solventar dudas como para compartir experiencias y para ello el foro ha sido la herramienta de comunicación más utilizada.

Como reflexión final, decir que no hemos entrado en el debate sobre la pertinencia y/o legitimidad de que un máster sea on-line o presencial, únicamente hemos analizado el uso que el alumnado ha realizado sobre las herramientas virtuales para su formación en el máster que nos compete, por entender que las TIC son recursos de enorme valor en ámbitos educativos, que posibilitan nuevos escenarios de enseñanza-aprendizaje y que los formadores debemos conocer como los usuarios los utilizan para así, aumentar las posibilidades que nos brindan dichos entornos educativos.

\section{FUENTES BIBLIOGRÁFICAS Y WEBGRÁFICAS.}

ARDILLA, M. (2011). Indicadores de calidad de las plataformas educativas digitales Educ.Educ. Vol. 14, No. 1, 189-206.

BALLESTEROS, C.; CABERO, J.; LLORENTE, Ma C. \& MORALES, J.A. (2010). Usos del e-learning en las universidades andaluzas: Estado de la situación y análisis de buenas prácticas. Pixel-Bit. Revista de Medios y Educación. № 37 pp. 7- 18.

BERGIEL, B.; BERGIEL, E. \& BALSMEIER, P. (2008). Nature of virtual teams: a summary of their advantages and disadvantages, Management Research News, Vol. 31, 99-110.

CABERO, J. (2006). Bases pedagógicas del e-learning. Revista de Universidad y Sociedad del Conocimiento. Vol. 3 - N. 1 http://rusc.uoc.edu/ojs/index.php/rusc/article/view/v3n1cabero $(11 / 03 / 11)$.

CASTELLS, M. (2010). The Rise of the Network Society: The Information Age: Economy, Society, and Culture, Oxford, UK: Blackwell Publishing.

CEDEP. Centro de Estudio de Postgrado de la Universidad Pablo de Olavide. Oferta formativa. Máster de Educación para el Desarrollo, Sensibilización Social y Cultura de Paz (II Edición). http://www.upo.es/postgrado/ (12/02/2011).

Declaración del Milenio (2000). http://www.un.org/spanish/milenio/ares552.pdf (12/02/2011).

Declaración Universal de los Derechos Humanos

http://www.un.org/es/documents/udhr/index.shtml (21/03/11). 
DUART, J. (2011). La internacionalización de la universidad en la sociedad red. RUSC. Revista de Universidad y Sociedad del Conocimiento, América del Norte, 812072011.

El Espacio Europeo de Educación Superior-Alcanzando las metas. Comunicado de la Conferencia de Ministros Europeos responsables de Educación Superior. Bergen, 19-20 de Mayo de 2005. http://www.eees.es/pdf/Bergen ES.pdf (12/02/2011).

FANDOS, M. (2006). El reto del cambio educativo: nuevos escenarios y modalidades de formación. Educar, 38, 243-258.

GARCÍA DEL DUJO, A. \& SUÁREZ GUERRERO, C. (2011). Interacción virtual y aprendizaje cooperativo: un estudio cualitativo. Revista de Educación. 354, 473-498.

GIL, A. F. \& ROCA-PIERA, J. (2011). Movilidad virtual, reto del aprendizaje de la Educación Superior en la Europa 2020. RED. Revista de Educación a Distancia, 26, 1-16.

GONZÁLEZ, A.D.; ACOSTA, Y. \& MOYARES, Y. (2010). Propuesta de un manual de usabilidad y accesibilidad para el desarrollo de personalizaciones de la plataforma de teleformación en Moodle. EDUTEC, Revista Electrónica de Tecnología Educativa. Núm. 34 Recuperado el 20/06/2011 http://edutec.rediris.es/Revelec2/Revelec34/desarrollo manual usabulidad accesibilidad personalizaciones moodle.html.

LAVE, J. \& WENGER, E. (2003). El aprendizaje situado. México: Universidad Nacional Autónoma de México.

OSLER, A. (1994). Development Education. Global perspectives in the curriculum. Londres-Nueva York: Cassell.

RAMOS, S. J.; DE LA OSA, J. F. \& DE TORO, F. J. (2009). Una plataforma para gestión de clases virtuales interactivas. EDUTEC, Revista Electrónica de Tecnología Educativa. Núm. 28 Recuperado el 07/08/2011 de http://edutec.rediris.es/Revelec2/revelec28/edutec28_plataforma_gestion_clases virtuale s.html

SÁNZHEZ RODRÍGUEZ, J. (2009): Plataformas de enseñanza virtual para entornos educativos. PixelBit. Revista de Medios y Educación. № 34 pp. 217-233.

\section{Para citar este artículo:}

TORRES, L., PRIETO, E. \& LÓPEZ, L. (2012). Entornos virtuales de enseñanza-aprendizaje. Evaluación del uso de las herramientas virtuales en el máster de educación para el desarrollo. Edutec, Revista Electrónica de Tecnología Educativa, 39. Recuperado el dd/mm/aa de http://edutec.rediris.es/Revelec2/Revelec39/evea_evaluacion_uso herramientas virtuales maste $\underline{\text { r.html }}$ 